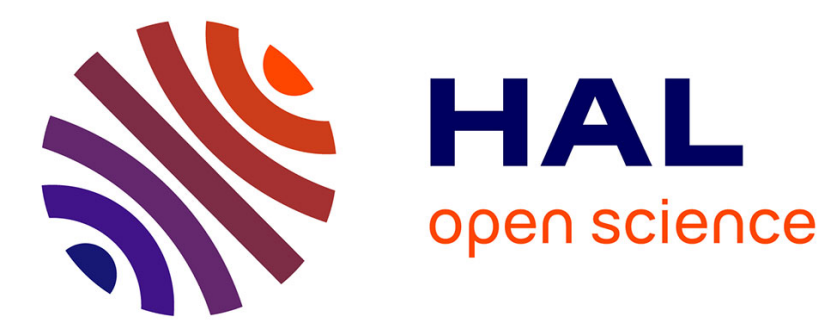

\title{
A Clustering-based Approach to Segment a Pavement Markings Line
}

\author{
Maxime Redondin, Laurent Bouillaut, Dimitri Daucher
}

\section{To cite this version:}

Maxime Redondin, Laurent Bouillaut, Dimitri Daucher. A Clustering-based Approach to Segment a Pavement Markings Line. International Journal of Performability Engineering, 2020, 16 (10), pp 1497-1508. 10.23940/ijpe.20.10.p1.14971508 . hal-03079410

\section{HAL Id: hal-03079410 https://hal.science/hal-03079410}

Submitted on 17 Dec 2020

HAL is a multi-disciplinary open access archive for the deposit and dissemination of scientific research documents, whether they are published or not. The documents may come from teaching and research institutions in France or abroad, or from public or private research centers.
L'archive ouverte pluridisciplinaire HAL, est destinée au dépôt et à la diffusion de documents scientifiques de niveau recherche, publiés ou non, émanant des établissements d'enseignement et de recherche français ou étrangers, des laboratoires publics ou privés. 


\title{
A Clustering-based Approach to Segment a Pavement Markings Line
}

\author{
Maxime Redondin ${ }^{\mathrm{a}, *}$, Laurent Bouillaut ${ }^{\mathrm{b}, *}$, and Dimitri Daucher ${ }^{\mathrm{c}}$ \\ ${ }^{a}$ VEDECOM Institute, 23 bis Allée des Maronniers, mobiLAB, F-78000 Versailles, France. \\ ${ }^{b}$ Université Paris-Est, Grettia (IFSTTAR), F-7755 Marne-la-vallée, France \\ ${ }^{c}$ Université Paris-Est, Lepsis (IFSTTAR), F-7755 Marne-la-vallée, France
}

\begin{abstract}
The maintenance of road infrastructure is a classic social challenge, especially in the context of a decreasing maintenance budget and the advent of autonomous vehicle traffic. Road markings need an accurate replacement strategy to guarantee that the markings remain perceptible. The retroreflective luminance of markings is currently dynamically quantifiable only by using a retroreflectometer such as the Ecodyn from MLPC. The main objective of this research is to construct a performance-based approach for retroreflective marking replacement adapted to a given road network. This approach involves three main tasks: localize the strategic area based on past inspections, determine an adapted decay model for a given area, and evaluate the economic impact of replacing markings. This paper focuses on the first task. We apply the Agglomerative Hierarchical Clustering (AHC) method to a given dataset to obtain a suitable markings line segmentation. Markings whose retroreflective luminance exhibits similar evolution over time are interpreted to belong to a specific area of the road network. When no follow-up replacement has occurred, a replacement detector deduces the date at which markings were laid from the clusters. The broken center line of the French National Road 4 illustrates the proposed approach; the road is divided into 5 clusters and 34 lifecycles. A study of markings laid in 2008 and replaced in 2012 shows important variations in the decay of the retroreflective luminance as identified by the clustering approach. Even for a single road, an optimal replacement strategy for retroreflective road markings is necessary and is composed of several local maintenance strategies.
\end{abstract}

Keywords: pavement marking; retroreflection luminance; Agglomerative Hierarchical Clustering

(Submitted on April 12, 2020; Revised on June 15, 2020; Accepted on September 16, 2020)

(C) 2020 Totem Publisher, Inc. All rights reserved.

\section{Introduction}

The maintenance of one given road infrastructure is a classic social challenge, especially in the context of a decreasing maintenance budget and the advent of autonomous vehicle traffic. The first motivates the development of an original maintenance strategy according to a given budget over several years. The second will define different maximum degradation thresholds on the pavement, the surface signing, and road equipment. An autonomous vehicle employs several different sensors to analyse the road on which it travels. VEDECOM chooses a multi-agent approach based on a camera [1]. A reliable marking line is more than $95 \%$ detected with fewer than $2 \%$ of false alarms [1]. Applied on a degraded road infrastructure, $85 \%$ of the emergency line is correctly detected and the center line is not seen by the system [2]. A global preventive maintenance strategy based on given degradation thresholds represents a solution to solve these two issues. A preventive replacement strategy that is able to guarantee perceptible pavement markings is an adapted solution to optimize lane detection. A clear road surface marking is more important than a pavement in good condition for an autonomous vehicle guidance system. However, up to now most efforts have been focused on pavement quality only. For example, Wang et al [3] compares different stochastic processes to model road fatigue cracking and Zouch et al [4] proposes a condition-based imperfect maintenance of the wearing course.

An autonomous vehicle may inspect the road infrastructure and more specifically, inspect pavement markings during its journey. Nowadays, no example of a marking line regularly inspected by an autonomous vehicle is known. The current

\footnotetext{
* Corresponding author.

E-mail address: maxime.redondin@vedecom.fr
} 
method for evaluation of marking degradation is based on the retroreflection luminance (from vehicle headlights). This strictly positive integer variable is measured in millicandela per square meter and by lux $\left(\mathrm{mcd} / \mathrm{m}^{2} / \mathrm{lx}\right)$. It is the only dynamically quantifiable measure and has become the only measure surveyed to evaluate marking degradation and its evaluation is subject to the strict conditions described in the NF EN 1436 rules [5].

Figure 1(a) illustrates the perception of a marking by nighttime according to their retroreflection luminance. For waterborne markings, this property is guaranteed by glass spheres mixed into the paint during application. A high level ( $>$ 150) guarantees a visible white marking. Their main degradation over time is induced by traffic, environmental conditions, and the material properties of markings and pavements. Markings progressively lose paint and glass beans, causing their retroreflective luminance to decrease over time. These results in a below-threshold level $(<150)$ and a visible grey object in the best case. When a marking's retroreflectivity has degraded sufficiently, replacement is the only possible maintenance action. European infrastructures require a minimum threshold of $150 \mathrm{mcd} / \mathrm{m}^{2} / \mathrm{lx}$ for a new marking [5]. Marking retroreflectivity can be dynamically inspected using a retroreflectometer such as the Ecodyn from MLPC. A retroreflectometer is fitted to the side of a car and connected to a laptop inside the car (Figure 1(a)). A white light is shone on the markings (Figure 1(b): "Emission") and the quantity of the retro-reflected light is measured (Figure 1(b): "Reception"). Associated software automatically creates a database in which each measurement is associated with a reference location (a kilometric point for example). The standard configuration analyses the retroreflective luminance every $40 \mathrm{~cm}$ but stores only the average over $100 \mathrm{~m}$.

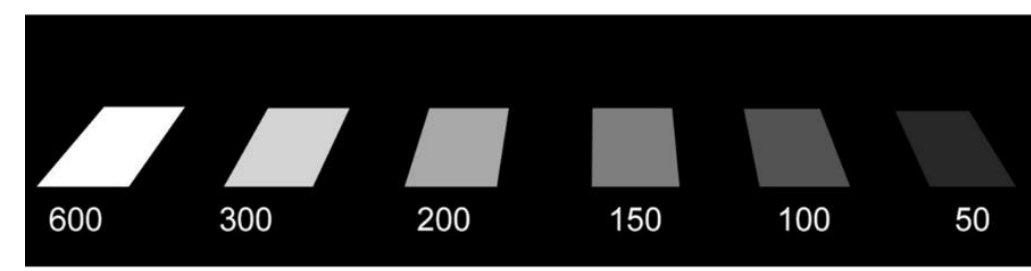

(a)

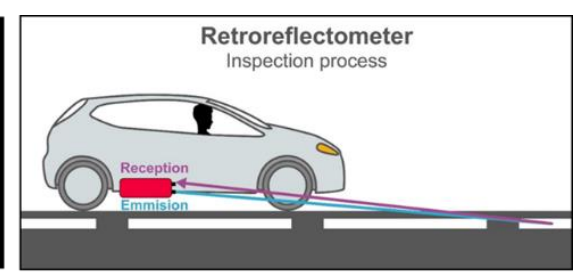

(b)

Figure 1. (a) Perception of a white marking according to it retroreflection luminance ( $\left.\mathrm{mcd} / \mathrm{m}^{2} / \mathrm{lx}\right)$; (b) Inspection process

Several degradation models exist in the current literature that are mainly based on a regression model. Two common weaknesses are observed: they are difficult to apply directly to a given road network and they suppose an available followup of the replacement. A single line is also not systematically replaced in its entirety. For logistic and safety reasons, road managers maintain only specific areas at a time. A first task for a surface marking replacement strategy is to identify strategic areas. A follow-up of the replacement is necessary to identify all the lifecycles. The constitution of feedback data is therefore key to developing a degradation or a lifetime model and finally deducing an adapted preventive replacement strategy. Thus, the main objective of this study is to construct a clustering-based approach for retroreflective marking replacement that is adapted to a given lane. This approach involves three tasks: localize strategic areas based on a feedback database, determine an adaptive lifetime model for the given area, and evaluate the economic impact of marking replacement. This paper focuses on the first task and proposes a clustering approach to localize the strategic areas.

First, Section 2 presents a state of the art main degradation model for pavement retro-reflective markings. Such models provide a prediction of failure occurrences and a preventive replacement is engaged before this instant. Then, Section 3.1 proposes a segmentation of one given line using an Agglomerative Hierarchical Clustering (AHC) approach [6]. Markings with similar retroreflective luminance evolution over time are identified and each cluster is interpreted with respect to a specific area of the road network. Section 3.2 completes this approach with a replacement detector to estimate the date at which markings were laid. The detector searches for a median retroreflection luminance gain between two inspections. In Section 4, an illustration of this approach is proposed, using a part of the broken centerline of the French National Road 4 (NR4). The surface signing is inspected once a year, but replacement follow-up is not available. However, every replacement campaign for marking is done in March. This situation runs counter to the literature, which assumed monthly road network inspections and completed follow-ups of required replacement. The road is divided into 5 clusters with 34 lifecycles. The study of markings laid in March 2008 and replaced in March 2012 shows that even on the same line and under the same cycle, important variations in the degradation of retroreflection luminance occur. The proposed clustering approach can isolate each different degradation mode. Finally, this example shows that even for a single road, an optimal replacement strategy for retroreflective road markings consists of several local maintenance strategies.

\section{State of the Art of Current Degradation Model of Retroreflective Marking}

The two main research fields regarding pavement markings are its detection by visibility sensors and its degradation. Hillel 
et al [7] presents research on lane detectors. The preferred sensor is a camera used with a clustering process able to extract markings from each image. The VEDECOM Institute proposed a multi-agent approach previously presented [1-2]. Independently: Xiao et al [8] and Lee et al [9] propose different options based on cameras. Kumar et al [10] proposes a solution based on a LIDAR and a cartographical approach is proposed by Hazelhoff et al [11]. A basic idea is to consider a lane detector as an inspection process. For example, Costello et al [12] proposed an inspection policy by camera and introduced five degradation levels. A stochastic maintenance strategy based on this model. However, this is a non-standard strategy and no example of a feedback database created by a lane detector is known. The current inspection process is based on a retroreflectometer and current degradation models evaluate the retroreflection luminance. This variable is explained by a regression method as a function of time, traffic, marking material, or lateral locations of markings. Such models provide a prediction of failure occurrences and a preventive replacement is engaged before this instant. A recent development is proposed by Owusu et al [13]. This paper presents the main degradation models currently used to evaluate retro-reflective markings.

The age of markings variables is present in all such models. It is also the only variable used on the exponential model proposed by Lu [14], linear models proposed by Scheuer et al [15], Taek et al [16], Sarasua et al [17] and Thamizharasan et al [18], and the inverted quadratic equation model proposed by Bahar et al [19]. Abboud and Bowman [20], Lindly and Wijesundera [1], and Lee et al. proposed independently the Vehicle Exposure $(V E)$ variable defined as a weighted product of the Annual Average Daily Traffic $(A A D T)$ and the age of markings. Each one proposed a logarithmic model function of the $V E$. Road infrastructure characteristics such as the traffic or the locations of markings are also considered by Karwa and Donnell [21] or Sitzabee et al [22]. The first proposed a multi-layer perceptron. The second proposed a multilinear model optimized to thermoplastic markings and is a function of time, the initial retroreflection value, the $A A D T$, the lateral locations of markings, and marking color. This model admits an alternative for waterborne markings and the number of snowplow transits is added by Mull and Sitzabee [23].

At least three papers bring into question a degradation model based on a regression technique. Ozelim and Turochy [24] recommend only models proposed by Sitzabee et al. [22] and Thamizharasan et al. [18]. Migletz et al. [25] and Kopf [26] show an important variation between models attributed to the road design, the marking material, the laying technique and the winter maintenance strategy. Some alternatives exist. Andrady [27] proposed an estimator of the useful lifetime of a retroreflective marking. Sathyanarayanan et al. [28] predicts the failure of given markings with a Weibull distribution. Asdrubali et al. [29] proposed the first known preventive replacement strategy for urban markings while respecting the NF EN 1436 rules [5]. Chimba et al [30] proposes a different degradation model based on a Markov chain.

Presented models are based on various assumptions. For example, Sitzabee et al [22] suggested an inspection after each replacement action, a follow-up of the replacement to estimate the age of markings, its material, one description of the road infrastructure, and an estimation of the $A A D T$. Thus, this model is not adapted to a retroreflective line yearly inspected where its follow-up of the replacement is not available. Operationally, proposed degradation models must be applied directly on the whole line. This assumption doesn't consider an eventual local degradation process or different maintenance strategies on the same road infrastructure. For example, this paper presents a road infrastructure segmented into open country roads, suburban areas, bypass, and a short highway. The proposed clustering-based approach consists of first segmenting a line into different sections correlated to one specific local infrastructure. Each one is then interpreted as a replacement strategic area and admits its own evolution of the retroreflection luminance but also its own replacement strategy. This last property could then be used to define an estimation of the follow-up of the replacement in respect of the NF EN 1436 rules [5] and be based on the median evolution of the retroreflection luminance. The Agglomerative Hierarchical Clustering (AHC) method [6] is chosen to segment a given line.

\section{Segmentation of a Markings Line by an AHC}

In this paper, any markings line is composed of $n>0$ retroreflectometer measures defined by $i=1, \cdots, n$. Each one is localized by a Point of Reference (PR). To simplify, one measure is analyzed as one pavement marking localized by a PR. The retroreflection luminance of marking $i$ at the inspection point $t$ is denoted $R L_{t}(i) \in N$. The whole monitoring of markings according to inspections $(T>1)$ realized is done by $I(1)$. One given marking $i \in I$ is described only by its $T>1$ inspection data. The monitoring is not completed by a description of the road infrastructure for example. A graphical representation of $I$ analyzes simultaneously the evolution of all markings from the first to the last inspection. A classic clustering approach, such as the AHC, identifies several clusters of markings that exhibit a similar retroreflection evolution over time. 


$$
I=\left\{\frac{\left(R L_{1}(i) ; \cdots, R L_{T}(i)\right)}{i=1, \cdots, n}\right\}
$$

\subsection{Identification of Strategic Areas}

The AHC interprets $I$ as vectors in the vectorial space $R^{T}$. Applying AHC results in sequences of partitions with increasing heterogeneity between partitions, resulting in $1<N<n$ clusters in which data is first isolated and then partitioned into clusters that include all similar objects [6]. General steps of the clustering process are: 1) each data point is initially assumed to be a cluster, 2) the distances between clusters are calculated, 3) the two closest clusters are merged, and 4) repeat from Step 2 until only one cluster remains containing all data.

Steps 1) and 3) do not present any difficulties. Step 2) introduces a distance measurement between two clusters. The Ward distance (2) is selected, where $d$ is the Euclidean distance. Here, $I_{x}$ and $I_{y}$ denote two clusters; $\underline{I_{x}}$ and $\underline{I}_{\underline{y}}$ are their average profiles; $\# I_{x}$ and $\# I_{y}$ represent their numbers. Various choices for distance measurements exist, such as the minimum or median distance [6]. The Ward distance merges the two clusters that cause the smallest decrease in betweenclass inertia. In fact, the Ward distance is defined as the reduction in the loss of between-class inertia between two clusters. Step 4) introduces the need for a stopping criterion. The classic approach is to represent the whole clustering process by an associated dendrogram. As shown in figure 5, this graphic is specific to the AHC and indicates the Ward distance between two given clusters. A classic stopping criterion is cutting the dendrogram when a compromise between a few clusters and a large average inter-cluster distance is found. A double cut is proposed in this paper. The first cut is empirically optimized according to geographical and logistical considerations. Indeed, to accumulate many clusters is logistically difficult to maintain for safety reasons. To control the quality of the cut, clusters may be projected on a road map. Consider now $N \geq 2$ clusters associated to the first cut denoted by $I_{1}, \cdots, I_{N}$.

$$
d_{w}\left(I_{x} ; I_{y}\right)=\frac{d\left(\underline{I_{x}} ; \underline{I_{y}}\right)}{\frac{1}{\# I_{x}}+\frac{1}{\# I_{y}}}
$$

When no follow-up of the replacement has occurred, marking deposition dates must be estimated. A replacement detector is proposed and is based on three observations: the retroreflection luminance decreases over time, a sudden gain in retroreflection luminance signals that a replacement occurred and the AHC tends to identify important gains in retroreflection luminance. A second cut of the dendrogram looks for a partition of sub-clusters defined only by some replacement actions. Sub-clusters created by this second cut are denoted by $I_{1}, \cdots, I_{M}$ where $M \geq N$.

\subsection{Identification of Replacement Action}

The proposed detector compares systematically two inspections on every sub-cluster $I_{1}, \cdots, I_{M}$. Consequently, it is unable to estimate replacement before the first inspection and estimated deposition dates are defined between two inspections. According to the NF EN 1436 [5], a minimum of $150 \mathrm{mcd} / \mathrm{m}^{2} / \mathrm{lx}$ is selected to represent a new marking. For a given cluster $I_{j} \forall j=1, \cdots, M$, five maintenance actions can be identified:

- A Full Replacement $(F R)$ operation involves a replacement of all the markings.

- A Full Stressed Replacement (FSR) operation involves a replacement of all the markings but the initial or the sudden gain retroreflection luminance is relatively low. An over maintenance action is an example.

- A Partial Replacement $(P R)$ operation consists of replacing only a part of the markings.

- A punctuated Replacement $(p R)$ operation consists of replacing only a part of the sub-cluster.

- A maintenance operation of Nothing $(\varnothing)$ is applied when no maintenance operation was conducted.

Let $A=\{F R, F S R, P R, p R, \emptyset\}$ be the set of possible replacement actions. The action detected for a marking $i$ during the inspection $\mathrm{t}$ is denoted as $A_{t}(i)$. For all inspections and all clusters, the number of replaced markings is counted. As shown in Figure 2, the replacement detector is based on two steps: the marking step and the clusters step.

The marking step analyzes whether the marking was replaced between inspections $t$ and $t-1$ (Figure 2(a)). The variation between inspections $t$ and $t-1$ is done by $\Delta R L_{t}$ (4). If $\Delta R L_{t}(i)>100$ and $R L_{t}(i)>150$ then the marking was replaced, and $A_{t}(i)=p R$. This step cannot determine whether this replacement action was isolated or is part of a wider 
replacement campaign. An increasing value of (at least) $100 \mathrm{mcd} / \mathrm{m}^{2} / \mathrm{lx}$ indicates a replace marking and can also be used to anticipate the degradation during the period between the deposition date and the inspection.

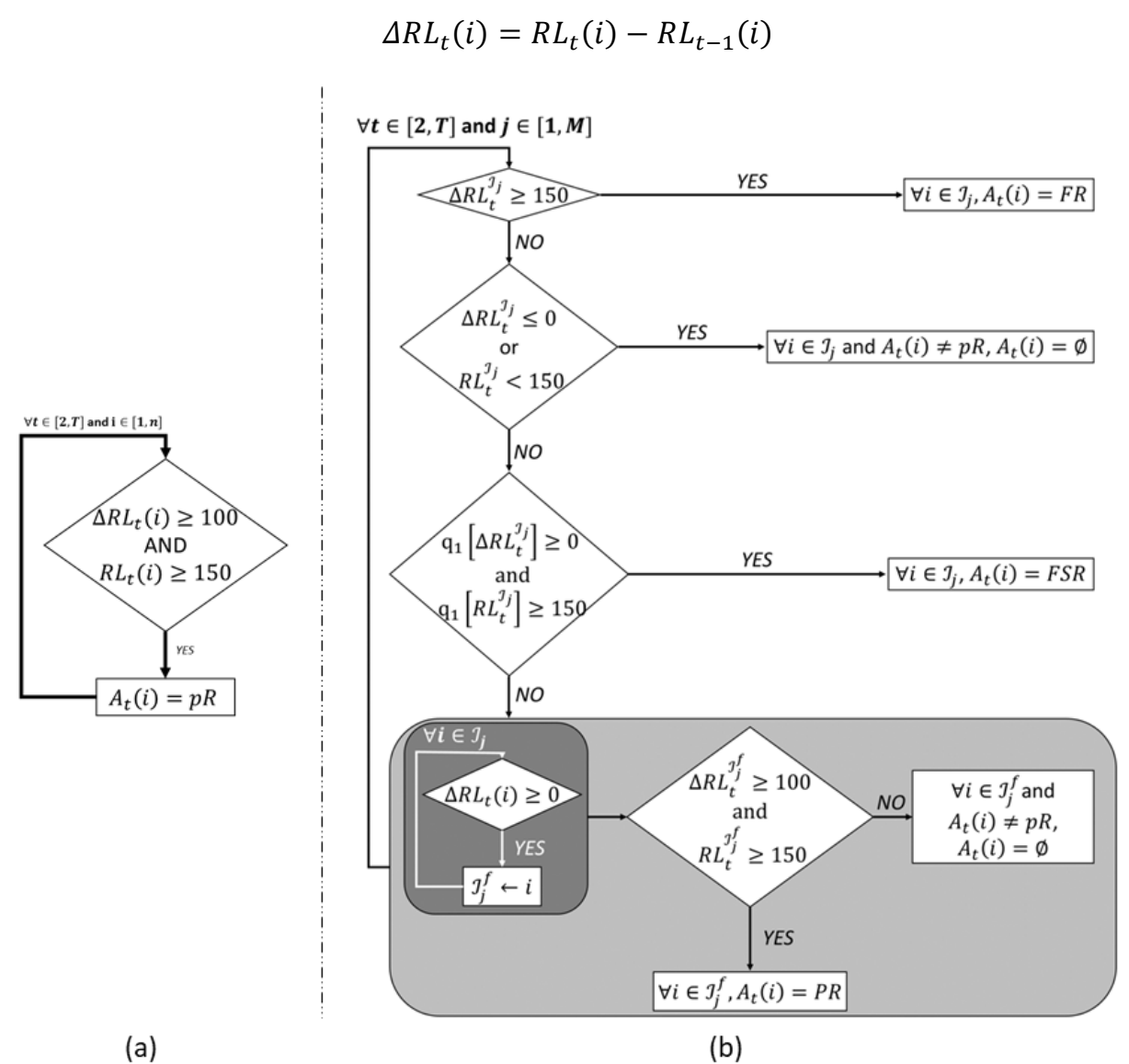

(a)

(b)

Figure 2. (a) The marking step to estimate marking deposition date; (b) The clustering step to estimate marking deposition date

Let us consider a given sub-cluster $I_{j}$ at inspection $t$. The cluster step analyzes whether the cluster was partially or completely replaced between $t$ and $t-1$ (Figure 2(b)). The process is split into four operations. Let us suppose $\Delta R L_{t}^{I_{j}}$ to be the median retroreflection luminance variation between inspections $t$ and $t-1$ observed on the sub-cluster $I_{j}$. If $\Delta R L_{t}^{I_{j}}>$ 150, then the cluster was fully replaced: $\forall i \in I_{j}, A_{t}(i)=F R$. This step detects all clear replacements.

$$
\Delta R L_{t}^{I_{j}}=\frac{1}{2}\left[\Delta R L_{t}\left(\frac{\# I_{j}}{2}\right)+\Delta R L_{t}\left(\frac{\# I_{j}}{2}+1\right)\right]
$$

Let us suppose $R L_{t}^{I_{j}}$ to be the median retroreflection luminance at inspection $t$ observed on the sub-cluster $I_{j}$. If $\Delta R L_{t}^{I_{j}}<0$ and $R L_{t}^{I_{j}}<150$, then the cluster was not replaced: $\forall i \in I_{j}$ and $A_{t}(i) \neq p R, A_{t}(i)=\emptyset$. This step meets replacement objectives: the cluster has a larger retroreflection luminance than is required by NF EN 1436 rules.

$$
R L_{t}^{I_{j}}=\frac{1}{2}\left[R L_{t}\left(\frac{\# I_{j}}{2}\right)+R L_{t}\left(\frac{\# I_{j}}{2}+1\right)\right]
$$

Let us suppose $q_{1}\left[\Delta R L_{t}^{I_{j}}\right]$ and $q_{1}\left[R L_{t}^{I_{j}}\right]$ are the first quartile of the retroreflective luminance between $t$ and $t-1$ and the first quartile of the retroreflective luminance at $t$ on $I_{j}$. If $q_{1}\left[\Delta R L_{t}^{I_{j}}\right]>0$ and $q_{1}\left[R L_{t}^{I_{j}}\right]>150$, then the cluster was fully replaced but the markings it contains are stressed: $\forall i \in I_{j}, A_{t}(i)=F S R$. By the first quartile definition, $75 \%$ of $I_{j}$ involves a gain of retroreflection luminance and the markings meet the NF EN 1436 rules. According to the first two operations, this gain is between 0 and $150 \mathrm{mcd} / \mathrm{m}^{2} / \mathrm{lx}$. An over replacement action is included in this situation. 


$$
\begin{aligned}
q_{1}\left[\Delta R L_{t}^{I_{j}}\right] & =\Delta R L_{t}\left(\frac{\# I_{j}}{4}\right) \\
q_{1}\left[R L_{t}^{I_{j}}\right] & =R L_{t}\left(\frac{\# I_{j}}{4}\right)
\end{aligned}
$$

Let's suppose $I_{j}^{f}(t)$ be the subset of $I_{j}$ to the markings where $\Delta R L_{t}>0$. This step constraints $I_{j}$ to the markings that involve a gain in retroreflection luminance. If $\Delta R L_{t}^{I_{j}^{f}(t)} \geq 100$ and $R L_{t}^{I_{j}^{f}(t)} \geq 150$, then $\forall i \in I_{j}^{f}(t), A_{t}(i)=P R$.

$$
I_{j}^{f}(t)=\left\{\frac{i \in I_{j}}{\Delta R L_{t}(i) \geq 0}\right\}
$$

\section{Presentation of the French National Road 4 Broken Center Line}

\subsection{Presentation of the French National Road 4}

A defined part of the broken center line of the French National Road 4 (NR4) is considered to illustrate the proposed approach. The road is presented by Figure 3. The NR4 runs between Paris and Strasbourg. The direction heading toward Strasbourg is chosen. Since 2007, the section between Courgivaux and Vauclerc $(\sim 102 \mathrm{~km})$ has been managed by the DIR Est. Pavement markings are yearly inspected in late September in collaboration with CEREMA Est. The selected retroreflectometer is an Ecodyn from MLPC which annotates each measure with a Point of Reference (PR). The surface signing is replaced according to inspections and the current maintenance budget in March. However, there is no follow-up of these replacement campaigns for markings. The same marking product is supposed to be fit. In this paper, only the feedback data base created by the Ecodyn is considered. Between 2007 and 2016, 970 markings were monitored (95\% of the considered NR4 section).

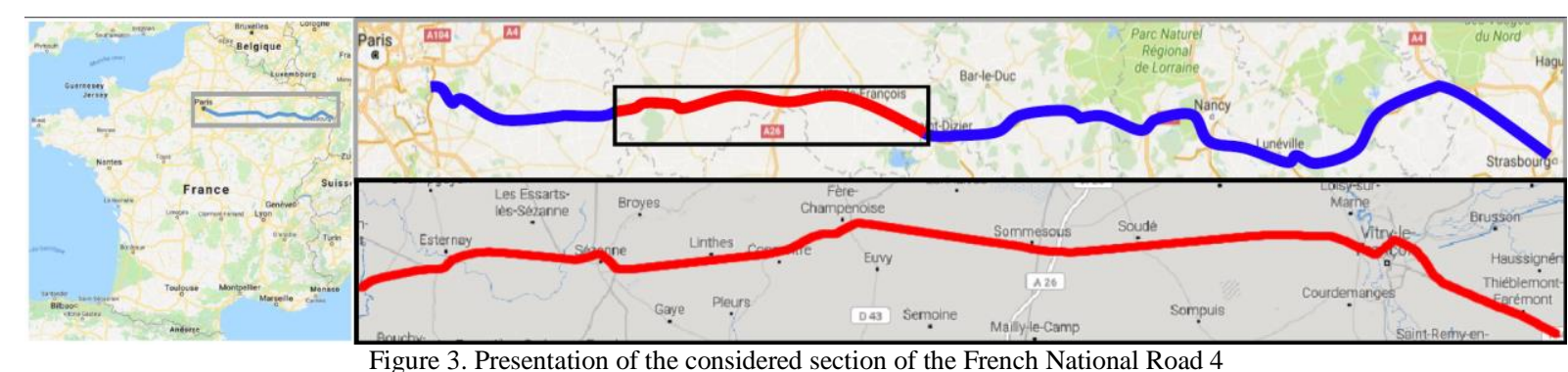

\subsection{Segmentation of the Broken Center Line}

Figure 4 introduces a cartographic AHC representation of a NR4 broken center line over time. Each cluster is represented by its median profile and is named based on the nearest city or by a remarkable observation. The follow-up of replacement is not available. From now, both the median variation and the median retroreflection identify possible complete replacement. The last line represents the whole broken center line and presents some general observations. Except in 2012, 2015 and 2016, the median retroreflection luminance is always under $150 \mathrm{mcd} / \mathrm{m}^{2} / \mathrm{lx}$. Two possible important replacement campaigns are observed in 2012 and 2015: $\Delta R L_{12}=105$ and $\Delta R L_{15}=173$. The longest decreasing period is observed between 2009 and 2011: $\Delta R L_{09}+\Delta R L_{10}+\Delta R L_{11}=-82$. The segmentation of the NR4 completes these observations.

(1) The cluster 1 corresponds to open country roads. It is the largest (43\% of markings) and shows a weak median retroreflection luminance between 2007 and 2011: $R L_{07}^{1}=103, R L_{08}^{1}=118, R L_{09}^{1}=103, R L_{10}^{1}=53, R L_{11}^{1}=55$. Two possible complete replacements are observed in 2012 and 2015: $\Delta R L_{12}^{1}=221, \Delta R L_{15}^{1}=260$.

(2) The cluster 2 is localized on different suburban areas. It is the second largest (35\% of markings) and shows the weakest median retroreflection luminance over time: $R L_{07}^{2}=96, R L_{11}^{2}=51, R L_{12}^{2}=120, R L_{13}^{2}=101$. One possible complete replacement is observed in 2015: $\Delta R L_{15}^{2}=158$.

(3) The cluster 3 is the bypass of Sézanne. This area was clearly replaced in 2011: $\Delta R L_{11}^{3}=529$ and $R L_{11}^{3}=577$. A 
strong degradation is also observed the next year: $\Delta R L_{12}^{3}=-406$. A possible complete replacement is observed in 2014: $\Delta R L_{14}^{3}=163$.

(4) The cluster 4 is the bypass of Fère-Champenoise. This area shows a good median retroreflection luminance over the time: $R L_{10}^{4}=160, R L_{13}^{4}=179, R L_{15}^{4}=365$. Four possible complete replacements are observed in 2009, 2012, 2014 and 2015: $\Delta R L_{09}^{4}=277, \Delta R L_{12}^{4}=75, \Delta R L_{14}^{4}=114$ and $\Delta R L_{15}^{4}=72$.

(5) The cluster 5 corresponds to a local free highway near the city of Sommesous. It is the smallest cluster (4\% of markings) and shows the strongest median retroreflection luminance over the time: $R L_{07}^{5}=826, R L_{08}^{5}=946, R L_{09}^{5}=510$, $R L_{14}^{5}=307$. Two possible complete replacements are observed in 2008 and 2014: $\Delta R L_{08}^{5}=141$ and $\Delta R L_{14}^{5}=183$. An important decrease is observed between 2008 and 2010: $\Delta R L_{09}^{5}+\Delta R L_{10}^{5}=-760$.

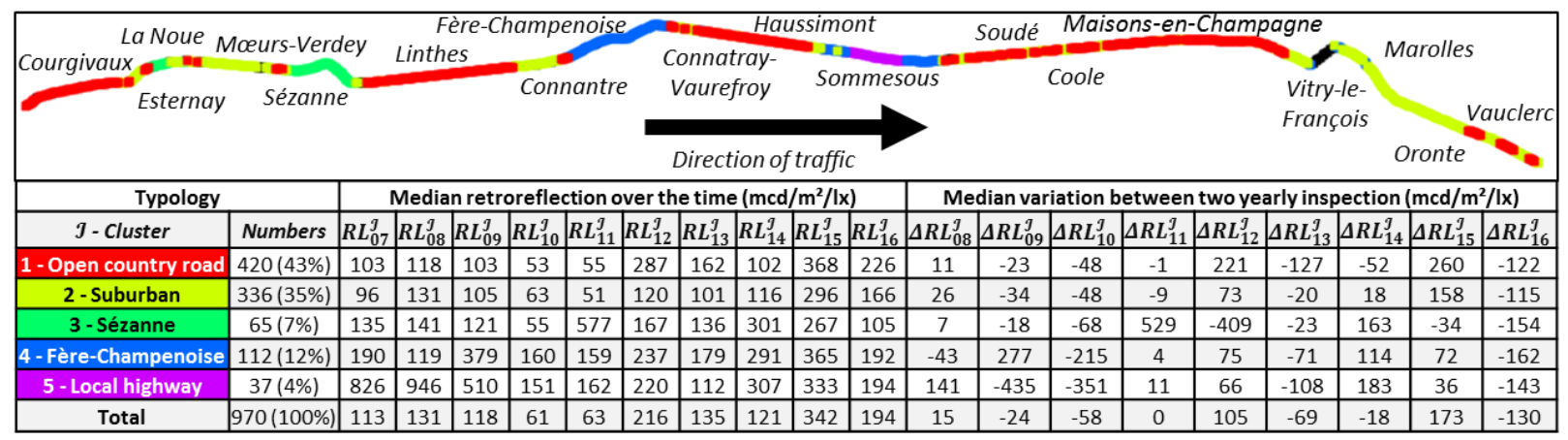

Figure 4. AHC representations of NR4 broken center line over time, showing the median profile of each cluster

The global analysis introduces two possible replacement campaigns in 2012 and 2015. This cartographical representation shows at least three additional pieces of information. First, the line is segmented into five clusters that are heterogeneous in size. Second, each cluster is correlated to some characteristic of the road infrastructure. Third, this segmentation identifies another possible replacement campaign. The two main examples are the clear complete replacement of Sézanne in 2011 and a possible replacement campaign on the three last clusters. However, this graphic does not represent the retroreflection monitoring and the clustering process remains to be verified.

Figure 5(a) shows the segmented monitoring of the broken center line between 2007 and 2016. Each cluster exhibits its own monitoring. For example, the local highway cluster shows a strong decrease between 2008 and 2010 ( -760 in median), a small increase between 2013 and 2015 (+219 in median) and the retroreflection luminance remains high over time. Otherwise, the Fère-Champenoise and local highway clusters have been similar since 2010 . Between 2007 and 2009, Fère-Champenoise presented clearly that two sub-clusters have been replaced in 2008 and 2009 respectively. Contrary to the previous analysis, different punctual replacements are identified in 2008 (cluster 2, 4 and 5), 2009 (cluster 4), and 2013 (cluster 1). Each inspection presents an important group which exhibits a low retroreflection luminance $\left(<150 \mathrm{mcd} / \mathrm{m}^{2} / \mathrm{lx}\right)$. Finally, the segmented monitoring confirms and completes the cartographical representation. However, at least one cluster could be divided into two: Fère-Champenoise presents two different evolutions between 2007 and 2010. Otherwise, the part replaced in 2008 is localized around the local highway. This observation could justify a finer segmentation of the line.

Figure 5(b) shows the dendrogram associated with the clustering process segmenting into five clusters. Two alternatives are identified. A cut into three clusters would join on the one hand the local highway and Fère-Champenoise and on other hand Sézanne and suburban areas. The current segmented monitoring justifies the merge between the local highway and Fère-Champenoise because their retroreflection luminance is similar since 2010. However, three distinct evolutions are presented between 2007 and 2010. Otherwise, Sézanne is clearly isolated on the map representation and focuses on an important replacement campaign in 2011. The second alternative proposes a cut into six clusters. According to the dendrogram, this sixth cluster is a distanced part of the open country road clusters. However, it corresponds to a discontinuous section of only $1.5 \mathrm{~km}$ and is defined by only one important replacement in 2015 .

Finally, the segmentation into five clusters seems to be a good compromise between limiting the number of clusters and producing clusters correlated to a specific characteristic of the road infrastructure. The replacement follow-up is not available and should thus be estimated. 


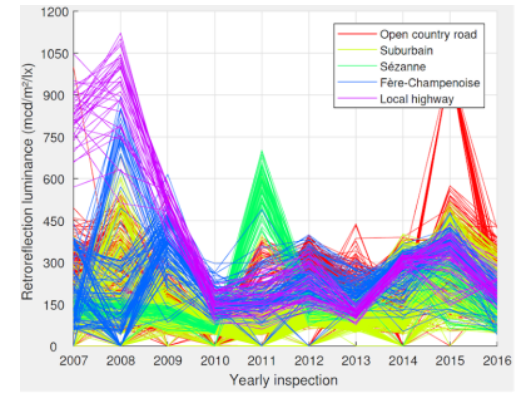

(a)

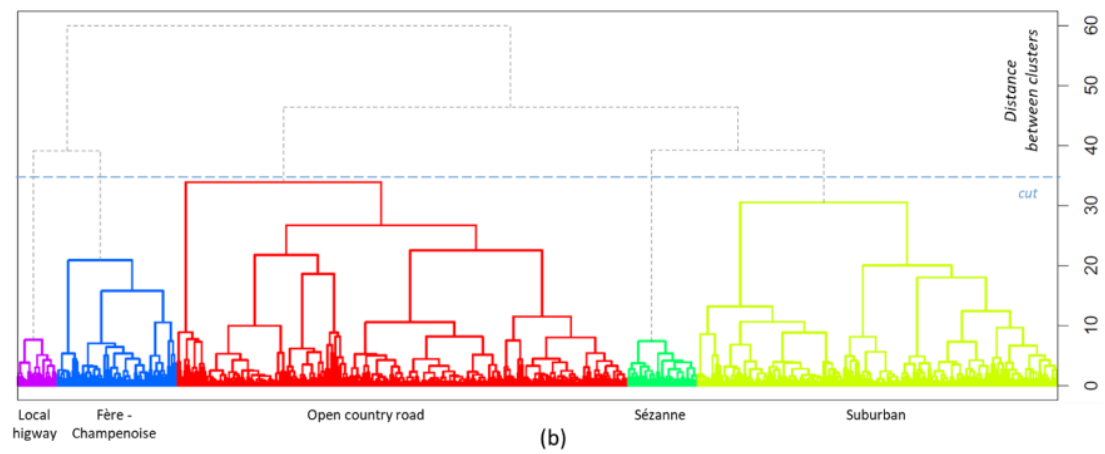

(b)

Figure 5. (a) Dendrogram associated with the AHC results of the NR4 broken center line; (b) Segmented monitoring between 2007 and 2016

\subsection{Estimation of the Broken Center Line Replacement Follow-up}

As presented in Figure 6, the second cut associated with the replacement detector is fixed at 15 clusters. This cut corresponds to a larger acceptable number of clusters. Contrary to the previous segmentation, sub-clusters are not necessarily correlated to a specific characteristic of the NR4: the sub-cluster 2 corresponds to the previous mentioned cluster 6 and is defined by a complete replacement in 2015, but the sub-cluster 10 corresponds to the bypass of Vitry-le-François. However, each one is associated with one cluster. Open country roads are composed of six sub-clusters: 1, 2, 3, 4, 5 and 6. Suburban areas are composed of four sub-clusters: 7, 8, 9 and 10. Sézanne is the sub-cluster 11. Fère-Champenoise is composed of three sub-clusters: 12, 13 and 14. The local highway cluster is the sub-cluster 15. Unsurprisingly, larger clusters are decomposed into several sub-clusters. On average, a given cluster is decomposed into 3 sub-clusters.

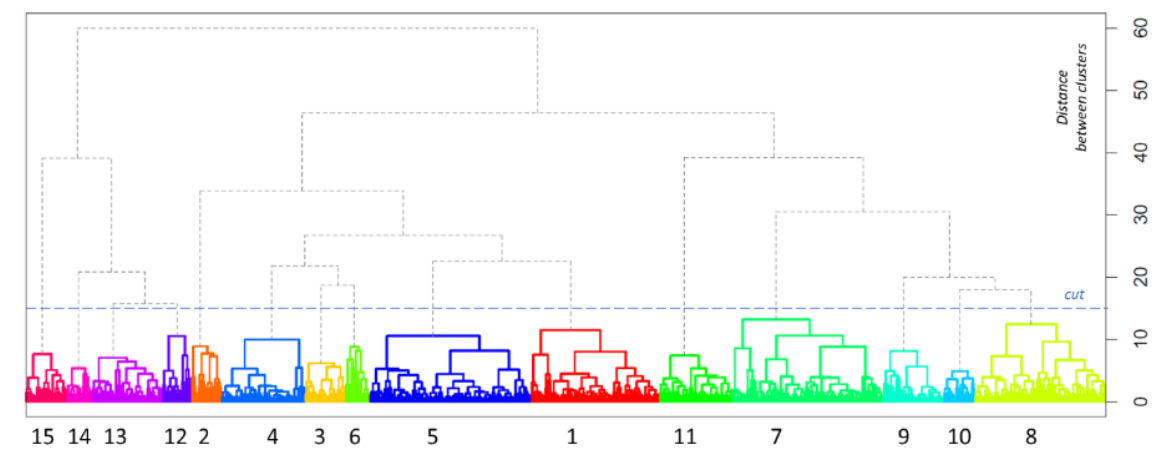

Figure 6. Dendrogram associated with the AHC results of the NR4 broken center line and associated median profile

Table 1 presents the follow-up of the replacement estimated using the replacement detector presented in Section 3.2. It indicates only the frequency of markings replaced. The last line is the whole broken center line. The two main replacement campaigns are estimated in $2012(59 \%)$ and $2015(77 \%)$. Few replacements are estimated in $2010(3 \%)$, $2013(3 \%)$ and $2015(<1 \%)$. Otherwise, the line benefits from targeted replacements. Each cluster is divided into its subclusters. For example, the open country road cluster is composed of 6 sub-clusters. Its sub-cluster 2 was partially replaced $(56 \%)$ in 2008 and completely replaced in 2015 . The whole cluster 1 was completely replaced in 2015 and no replacement was observed in 2010.

(1) Open country roads were completely replaced in 2015. Its sub-clusters exhibit on average 1.8 complete replacements since 2008. In 2012 (84\%), its three larger sub-clusters 1, 4 and 5 were completely replaced.

(2) Suburban areas have never been completely replaced. In 2014 (41\%), its larger sub-cluster 7 was completely replaced. The other sub-clusters are completely replaced next year (62\%). On average, sub-clusters exhibit 1.25 complete replacements since 2008. Three complete replacements are observed on the sub-cluster 10 (bypass of Vitry-le-François).

(3) Sézanne was completely replaced in 2011 and 2014.

(4) Fère-Champenoise was completely replaced in 2012 and 2015. Sub-clusters show on average 3.6 complete replacements since 2008. Most of the observed complete replacements are stressed replacements. The 2015 replacement corresponds mainly to an over-maintenance action due to an opportunist action (77\% of the whole line was replaced), in 
combination with a complete replacement the previous year. Other stress replacements are mainly attributed to the important retroreflection luminance observed on the figure 9 combined with a slow degradation process.

(5) The local highway was completely replaced in 2008, 2012, 2014. The 2008 stress replacement corresponds to an over-replacement action.

Table 1. Estimated follow-up of the replacement of the NR4 broken center line

\begin{tabular}{|c|c|c|c|c|c|c|c|c|c|c|}
\hline Cluster & Sub-cluster & 2008 & 2009 & 2010 & 2011 & 2012 & 2013 & 2014 & 2015 & 2016 \\
\hline \multirow{7}{*}{$\begin{array}{l}\text { Open country } \\
\text { road }\end{array}$} & 1 & $13 \%$ & $2 \%$ & & $3 \%$ & $100 \%$ & & $2 \%$ & $100 \%$ & $1 \%$ \\
\hline & 2 & $56 \%$ & $4 \%$ & & & $37 \%$ & $4 \%$ & $4 \%$ & $100 \%$ & \\
\hline & 3 & $11 \%$ & $6 \%$ & & $100 \%$ & $14 \%$ & & & $100 \%$ & \\
\hline & 4 & & $1 \%$ & & & $100 \%$ & & $12 \%$ & $100 \%$ & \\
\hline & 5 & $1 \%$ & & & $3 \%$ & $100 \%$ & & & $100 \%$ & \\
\hline & 6 & & & & $14 \%$ & $5 \%$ & $100 \%$ & $5 \%$ & $100 \%$ & \\
\hline & Total & $9 \%$ & $1 \%$ & & $12 \%$ & $84 \%$ & $5 \%$ & $3 \%$ & $100 \%$ & $<1 \%$ \\
\hline \multirow{5}{*}{ Suburban } & 7 & $29 \%$ & $7 \%$ & & $10 \%$ & $15 \%$ & & $100 \%$ & $4 \%$ & $1 \%$ \\
\hline & 8 & $6 \%$ & $7 \%$ & & $6 \%$ & $39 \%$ & $1 \%$ & $1 \%$ & $100 \%$ & \\
\hline & 9 & $9 \%$ & $11 \%$ & & $2 \%$ & $2 \%$ & & $2 \%$ & $100 \%$ & $2 \%$ \\
\hline & 10 & $100 \%$ & & $100 \%$ & & $7 \%$ & & & $100 \%$ & \\
\hline & Total & $23 \%$ & $7 \%$ & $8 \%$ & $7 \%$ & $21 \%$ & $<1 \%$ & $41 \%$ & $62 \%$ & $1 \%$ \\
\hline \multicolumn{2}{|c|}{ Sézanne } & & & & $100 \%$ & & & $100 \%$ & $11 \%$ & \\
\hline \multirow{4}{*}{$\begin{array}{c}\text { Fère - } \\
\text { Champenoise }\end{array}$} & 12 & $12 \%$ & $100 \%$ & $8 \%$ & $23 \%$ & $100 \%$ & $8 \%$ & & $100 \%$ & \\
\hline & 13 & & $100 \%$ & & & $100 \%$ & & $100 \%$ & $100 \%$ & \\
\hline & 14 & $100 \%$ & & & $13 \%$ & $100 \%$ & & $100 \%$ & $100 \%$ & \\
\hline & Total & $24 \%$ & $79 \%$ & $2 \%$ & $8 \%$ & $100 \%$ & $4 \%$ & $77 \%$ & $100 \%$ & \\
\hline \multicolumn{2}{|c|}{ Local highway } & $100 \%$ & & & & $100 \%$ & & $100 \%$ & $11 \%$ & \\
\hline \multicolumn{2}{|c|}{ Global } & $18 \%$ & $12 \%$ & $3 \%$ & $15 \%$ & $59 \%$ & $3 \%$ & $35 \%$ & $77 \%$ & $<1 \%$ \\
\hline
\end{tabular}

This summarized follow-up of the replacement shows that sub-clusters are built according to several complete replacement campaigns, occasionally supported by punctual replacement. For example, Sézanne was replaced twice since 2008. Larger clusters take advantage of targeted replacements. For example, the sub-cluster is included in the open country road cluster and is defined by only one important replacement in 2015 . This follow-up provides additional information on the current maintenance strategy. For example, an important replacement campaign was done in 2015 (77\%). This is the reason why no replacement was detected in $2016(<1 \%)$. A similar situation is presented in $2012(59 \%)$ and $2013(3 \%)$.

The replacement detector estimated 34 lifecycles between 2008 and 2016. Figure 7 presents the cycle of 92 markings deposited in May 2008 and replaced in May 2012. However, inspections are done once a year in September between 2008 and 2011. Retroreflective degradation clearly involves two steps. The first step, between 2008 and 2010 , is a fast-decay period (median $-625 \mathrm{mcd} / \mathrm{m}^{2} / \mathrm{lx}$ between 2008 and 2010). The second step shows a stable variation of $+14 \mathrm{mcd} / \mathrm{m}^{2} / \mathrm{lx}$. This pattern is in accordance with other reports in the literature from Sathyarayanan [27] or Sarazura [17]. This example clearly shows the differences in the degradation rate with respect to the clusters. The local highway clusters have the highest 
retroreflection luminance. The open country road and suburban clusters have the lowest retroreflection luminance. However, the degradation rate is more stable in the suburban cluster. The Fère-Champenoise cluster has an intermediate retroreflection luminance between 2008 and 2010. Within the same lifecycle, four different degradation rates were identified by the AHC.

Finally, the NR4 broken center line example shows that clusters are localized to areas such as a city, a bypass, and so on. The current maintenance strategy could be estimated from the segmentation. For example, the local highway of Sommesous is isolated in the segmentation. The identification of several strategic areas shows the difficulty in directly applying a single degradation model for retroreflective markings, even on a given single road. Each cluster degrades at a specific rate, and more broadly, requires its own replacement strategy. A preventive replacement strategy adapted to this line should comprise several local replacement strategies. AHC applied across all inspections was able to define the entire strategic area.

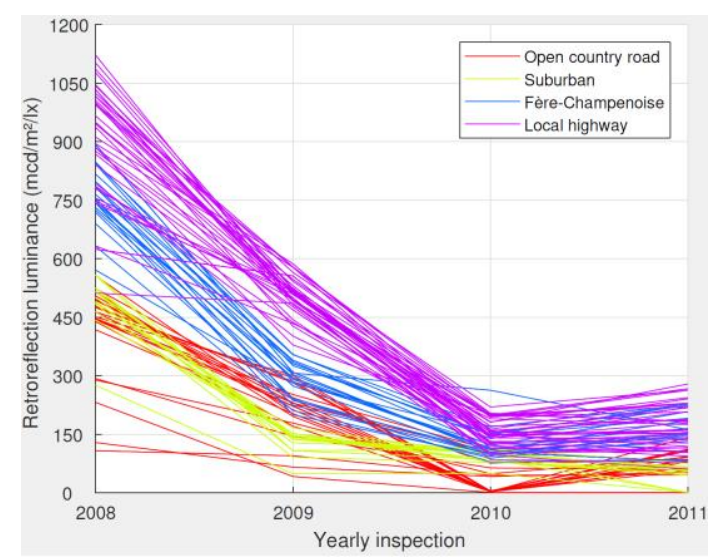

Figure 7. The 2008-2012 lifecycle segmented into the four presented clusters

\section{Conclusion}

Road infrastructure maintenance is a classic social challenge in the context of a decreasing maintenance budget. One important equipment to maintain are pavement markings which define traffic lanes. This is the reason why autonomous vehicle guidance systems include a lane detector. However, this option must be optimized by a preventive replacement strategy able to guarantee perceptible pavement markings and improve the quality and reliability of the road infrastructure. Currently, evaluation of degradation is based on retroreflection luminance. This measure evaluates the nighttime visibility and is dynamically quantifiable by a retroreflectometer such as the Ecodyn from MLPC.

The degradation is currently explained by a regression method as a function of time, traffic, or marking material. The associated preventive replacement strategy provides a prediction of failure occurrences. And finally, the replacement is engaged before this instant. However, these models present three main difficulties. First, the prediction quality shows an important variation from one model to another. Second, the follow-up of the replacement is known. Third, proposed degradation models must be applied directly on a whole line. However, a line is only observed by retroreflectometers and the follow-up of the replacement may not be available. Also, current assumptions do not consider an eventual local degradation process or different maintenance strategies on the same road infrastructure.

In this paper, the feedback database describes completely one given markings line and is generated by retroreflectometers. The monitoring over time of the retroreflection luminance is the first and only indicator to develop a preventive replacement strategy. A clustering approach simplifies the analysis. Each cluster presents one specific evolution over time, including each replacement action and be projected on a road map. Without additional information on the feedback database, the markings line is segmented into several maintenance strategic areas defined by both a specific evolution of the retroreflection luminance and a specific geographic position.

The Agglomerative Hierarchical Clustering (AHC) is chosen to segment the line. The clustering process is represented by a dendrogram which opens a discussion on the number of clusters to choose. This is the main reason why this approach was chosen. A double cut is proposed. The first cut segments a line into several sections correlated to one specific local infrastructure. The NR4 broken center line is segmented into five clusters. Each one is correlated to the road infrastructure: a bypass, a local highway or an open country road. 
This paper introduces the situation where the follow-up of the maintenance is not available. A replacement detector is based on the following two observations. First, each cluster of the first segmentation exhibits its own evolution of the retroreflection luminance as well as its own maintenance strategy. Second, the retroreflection luminance decreases over time. The median gain of retroreflection luminance between two inspections per cluster is an accepted indicator to estimate the follow-up of the replacement. This detector is optimized by a second cut of the dendrogram. Sub-clusters deduced from it correspond to the larger acceptable number of clusters and are empirically defined by several gains of retroreflection between two inspections. The final replacement detector is also based on different decisions in respect of the NF EN 1436 rules.

The detector estimates 34 lifecycles between September 2008 and September 2016. The current replacement strategy is now known. The bypass of Sézanne has been completely replaced two times since 2008, for example. Complete life cycles are extracted from the estimated follow-up of the replacement and are similar to those presented in the literature. This paper presents the cycle of markings laid in March 2008 and replaced in March 2012. This monitoring is also segmented into four clusters. Each one exhibits its own degradation process. For example, the retroreflection luminance is strong on the local highway but the degradation process is very fast. This example shows that the segmentation of the whole line is also represented on a given life cycle.

Three limits are identified on the proposed approach. First, the segmentation is not perfect. For example, the section between Soudé and Maisons-en-Champagne is mainly classified as open country roads. However, small sections of $1 \mathrm{~km}$ classified in suburban clusters are included and correspond to some small cities such as Coole. In future work, the authors suggest completing the feedback database with a description of the road infrastructure and the complete surface signing. A first approach based on a factorial analysis presents a thinner segmentation based mainly on the traffic lane organization. These first encouraging results motivate an extending study including the French Government Open Data. Second, the clustering process is empirically stopped by a comparative study between the associated dendrogram and the representation on a road map. An automatic stopping criterion is advisable. Finally, the proposed replacement detector must be compared to a line regularly inspected with a complete follow-up of the replacement. Obviously, this work is necessary to estimate the prediction level of the detector. An additional interest is to optimize different steps, which are currently mainly influenced by the NF EN 1436 [5].

This paper proposed an original clustering-based approach to segment a pavement markings line. Even for a single road, an optimal preventive replacement strategy for retroreflective road markings is necessary and is composed of several local maintenance strategies. A line is now a segment on a different replacement strategic area. Each one could be associated with one degradation model based on a regression method. In future work, the reliability approach proposed by Sathyanarayanan et al [27] may be preferred because it represents an opportunity to develop a stochastic preventive maintenance strategy for road markings.

\section{Acknowledgements}

VEDECOM Institute and IFSTTAR thank CEREMA Est and DIR Est for inspection data of the National Road 4.

\section{References}

1. M. Revilloud, D. Gruyer, and M. C. Rahal, "A Lanemarker Estimation Method for Improving Lane Detection," in Proceedings of the 19th IEEE International Conference on Intelligent Transportation Systems (ITSC), pp. 289-295, Rio de Janeiro, Brazil, November 2016

2. M. Revilloud, D. Gruyer, and M. C. Rahal, “A New Multi-Agent Approach for Lane Detection and Tracking," in Proceedings of the 2016 IEEE International Conference on Robotics and Automation (ICRA), pp. 3147-3153, Stockholm, Sweden, May 2016

3. Y. Wang, K. C. Mahboub, and D. E Hancher, "Survival Analysis of Fatigue Cracking for Flexible Pavements based on Longterm Pavement Performance Data," Journal of Transportation Engineering, Vol. 131, No. 8, pp. 608-616, August 2005

4. M. Zouch, T. Yeung, and B. Castanier, "A Condition-based Imperfect Maintenance Model with Action Dependent Deterioration," in Proceedings of the 2009 Industrial Engineering Research Conference (IIE), pp. 2158-2169, Miami, UnitedStates, June 2009

5. AFNOR, "NF EN 1436 + A1 - Road Marking Material - Road Marking Performance for Road Users," AFNOR Editions, January 2018

6. S. Tufféry, "Data Mining and Statistics for Decision Making," John Wiley \& Sons, 2001

7. A. B. Hillel, R. Lerner, D. Levi, and G. Raz, "Recent Progress in Road and Lane Detection: A Survey," Machine Vision and Applications, Vol. 25, No. 3, pp. 727-745, February 2012

8. L. Xiao, L. Chuanxiang, D. Zhao, T. Chen, and B. Dai, "Road Marking Detection based on Structured Learning," in Proceedings of the 12th World Congress on Intelligent Control and Automation Robotics and Automation (WCICA), pp. 2047- 
2015, Guilin, China, September 2016

9. S. Lee, J. Kim, J. Shin Yoon, S. Shin, O. Bailo, N. Kim, et al., "Vpgnet: Vanishing Point Guided Network for Lane and Road Marking Detection and Recognition," in Proceedings of 2017 IEEE International Conference on Computer Vision (ICCV), pp. 1947-1955, Venise, Italy, October 2017

10. P. Kumar, C. P. McElhinney, P. Lewis, and T. McCarthy, "Automated Road Markings Extraction from Mobile Laser Scanning Data," International Journal of Applied Earth Observation and Geoinformation, Vol. 32, pp. 125-137, October 2014

11. L. Hazelhoff, I. M. Creusen, and T. Woudsma, "Exploiting Automatically Generated Databases of Traffic Signs and Road Markings for Contextual Co-Occurrence Analysis," Journal of Electronic Imaging, Vol. 24, No. 6, November 2015

12. S. B. Costello, W. F. Moss, C. J. Read, and S. Grayer, "Life-Cycle Planning Methodology for Ancillary Highway Assets," Proceedings of the Institution of Civil Engineers-Transport, Vol. 164, No. 4, pp. 251-257, November 2011

13. V. Owusu, Y. A. Tuffour, D. A. Obeng, and M. Salifu, "Degradation of Retro-Reflectivity of Thermoplastic Pavement Markings: A Review," Open Journal of Civil Engineering, Vol. 8, No. 3, pp. 301-311, September 2018

14. J. J. Lu, "Performance of Traffic Markings in Cold Regions," Transportation Research Center, Institute of Northen Engineering, University of Alaska Fairbanks, 1995

15. M. Scheuer, T. L. Maleck, and D. R. Lighthizer, "Paint-Line Retroreflectivity Over Time," Transportation Research Record, Vol. 1585, No. 1, pp. 53-63, January 1997

16. J. Taek, T. L. Maleck, and W. C. Taylor, "Pavement Making Material Evaluation Study in Michigan," ITE Journal of Institute of Transportation Engineers, Vol. 69, No. 7, pp. 44-51, July 1999

17. W. A. Sarasua, D. B. Clarke, and W. J. Davis, "Evaluation of Interstate Pavement Marking Retroreflectivity," Report No. FHWA-SC-03-01 to South Carolina Department of Transportation, April 2003

18. W. A. Thamizharasan, W. A. Sarasua, D. B. Clarke, and W. J. Davis, "A Methodology for Estimating the Lifecycle of Interstate Highway Pavement Marking Retroreflectivity," in Proceedings of the 82nd Annual Meeting of the Transportation Research Board of the National Academies, Washington, DC, August 2002

19. G. Bahar, M. Masliah, T. Erwin, E. Tan, and E. Hauer, "Pavement Marking Materials and Markers: Real-World Relationship Between Retroreflectivity and Safety Over Time," National Cooperative Highway Research Program, Final Report Project 1728, April 2006

20. N. Abboud and B. L. Bowman, "Cost-and Longevity-based Scheduling of Paint and Thermoplastic Striping," Transportation Research Record, Vol. 1794, No. 1, pp. 55-62, January 2002

21. V. Karwa and E. Donnell, "Predicting Pavement Marking Retroreflectivity using Artificial Neural Networks: Exploratory Analysis," Journal of Transportation Engineering, Vol. 137, No. 2, pp. 91-103, January 2011

22. W. E. Sitzabee, J. E. Hummer, and W. Rasdorf, "Pavement Marking Degradation Modeling and Analysis," Journal of Infrastructure Systems, Vol. 15, No. 3, pp. 190-199, August 2009

23. D. M. Mull and W. E. Sitzabee, "Paint Pavement Marking Performance Prediction Model," Journal of Transportation Engineering, Vol. 138, No. 5, pp. 618-624, May 2012

24. L. Ozelim and R. E. Turochy, "Modeling Retroreflectivity Performance of Thermoplastic Pavement Markings in Alabama," Journal of Transportation Engineering, Vol. 140, No. 6, April 2014

25. J. Migletz, J. L. Graham, D. W. Harwood, and K. M. Bauer, "Service Life of Durable Pavement Markings," Transportation Research Record, Vol. 1749, No. 1, pp. 13-21, January 2001

26. J. Kopf, "Reflectivity of Pavement Markings: Analysis of Retroreflectivity Degradation Curves," Washington State Department of Transportation, Report No. WA-RD 592.1, September 2004

27. A. L. Andrady, "Pavement Marking Materials: Assessing Environment Friendly Performance," Transportation Research Record, Vol. 392, January 2001

28. S. Sathyanarayanan, V. Shankar, and E. T. Donnell, "Pavement Marking Retroreflectivity Inspection Data: A Weibull Analysis," Transportation Research Record, Vol. 2055, No. 1, pp. 63-70, January 2008

29. F. Asdrubali, C. Buratti, E. Moretti, F. D'Alessandro, and S. Schiavoni, "Assessment of the Performance of Road Markings in Urban Areas: The Outcomes of the CIVITAS Renaissance Project," Open Transportation Journal, Vol. 7, pp. 7-19, March 2013

30. D. Chimba, E. Kidando, and M. Onyango, "Evaluating the Service Life of Thermoplastic Pavement Markings: Stochastic Approach," Journal of Transportation Engineering, Part B: Pavements, Vol. 144, No. 3, pp. 1-8, May 2018 